\title{
Effect of Trastuzumab among HER2-Positive Breast Cancer Patients that Achieved Pathologic Complete Response after Neoadjuvant Chemotherapy
}

\author{
Xinguang Wang Yingjian He Zhaoqing Fan Tianfeng Wang Yuntao Xie \\ Jinfeng Li Tao Ouyang \\ Key Laboratory of Carcinogenesis and Translational Research (Ministry of Education/Beijing), Breast Center, Peking \\ University Cancer Hospital and Institute, Beijing, China
}

\section{Keywords}

Trastuzumab - Neoadjuvant therapy · Pathologic complete response · Adjuvant treatment · Survival

\begin{abstract}
Background: We sought to investigate the incremental benefit of trastuzumab in patients with HER2-positive breast cancer who achieved a pathologic complete response (pCR) after neoadjuvant chemotherapy (NACT). Methods: The data of HER2-positive invasive breast cancer patients treated with NACT and achieving pCR were obtained from the institutional database. Patients were categorized according to trastuzumab administration. The Kaplan-Meier method and log-rank estimates were used to test the association between trastuzumab administration and survival. Univariate and multivariate Cox regressions were used to obtain hazard ratios. Results: Of 223 patients, 83 (37.2\%) were treated with NACT without trastuzumab and 140 (62.8\%) were treated with NACT plus trastuzumab for 1 year. After a median follow-up of 67 months, the trastuzumab group showed improved relapse-free survival compared with the no-trastuzumab group (95.7 vs. $87.8 \%$, hazard ratio $=0.31, p=0.028$ ). No significant difference in distant disease-free survival or overall survival was observed ( $p=0.250$ and 0.432 , respectively). Multivariate analysis identified endocrine therapy and trastuzumab administration to be associated with decreased risk of relapse ( $p=0.018$ and 0.030 , respectively).
\end{abstract}

Conclusion: The administration of trastuzumab should be considered standard treatment for HER2-positive patients who have achieved pCR after NACT alone.

(c) 2019 S. Karger AG, Basel

\section{Introduction}

HER2 is amplified in about $15-25 \%$ of breast cancers depending on the criteria used to define HER2 positivity [1]. Adjuvant use of trastuzumab to treat HER2-positive breast cancer was approved by the US Food and Drug Administration in 2006 after clinical trials showed compelling evidence of favorable efficacy and safety profiles of trastuzumab. We are still searching for tools to effectively tailor treatment in order to achieve optimal risk/ benefit and cost/benefit ratios for patients with HER2positive disease. Pathologic complete response ( $\mathrm{pCR}$ ) after neoadjuvant chemotherapy (NACT) may be a potential surrogate. Neoadjuvant trials incorporating trastuzumab as well as a recent meta-analysis have shown that patients who achieve pCR after NACT have an excellent prognosis [2-5].

Little is known regarding the incremental benefit of trastuzumab after a pCR has been achieved. Hypothetically, trastuzumab administration might become relatively less important as the efficacy of chemotherapy increases [6]. Retrospective data from the MD Anderson 


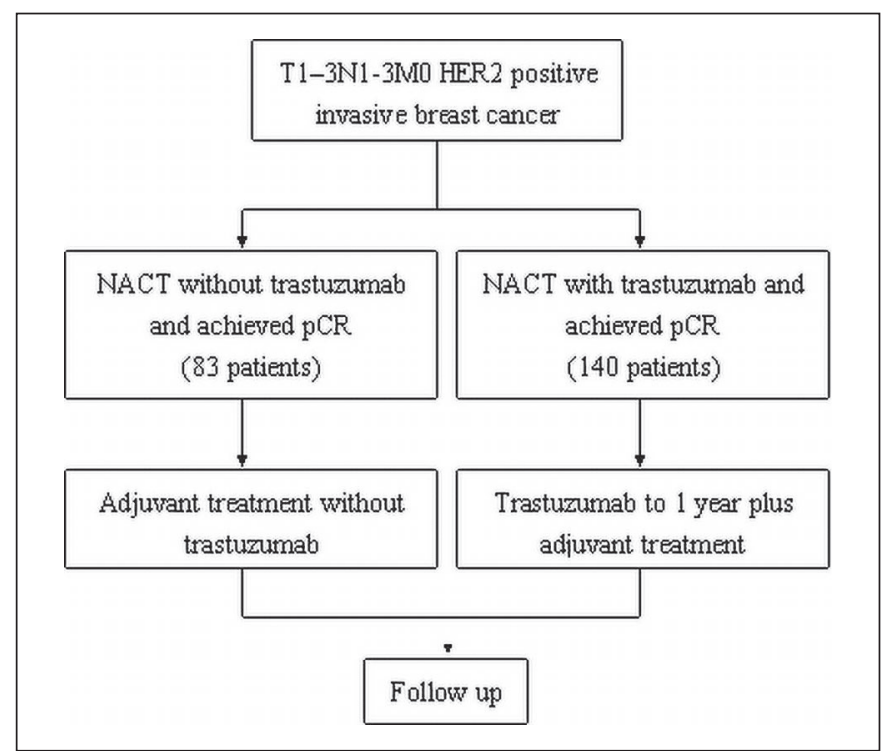

Fig. 1. Study design.

Cancer Center indicated that in HER2-positive breast cancer patients treated with trastuzumab-based NACT and having achieved pCR, adjuvant trastuzumab did not further improve overall survival (OS) or relapse-free survival (RFS) [7]. It was also believed that HER2-positive patients who achieved pCR after NACT without trastuzumab had a good prognosis, and the poorer prognosis of HER2-positive breast cancers as a group could be explained by a higher likelihood of relapse in those who do not achieve a pCR [8]. These results raise the question of the incremental benefit of systemic trastuzumab in pCR patients after NACT. More recently, the neoadjuvant NOAH trial showed an improved event-free survival in pCR patients in the trastuzumab group compared with pCR patients in the no-trastuzumab group [4]. We seek to contribute to this topic by reporting our patient outcomes.

\section{Patients and Methods}

\section{Patients}

A list of T1-3N1-3M0 patients with HER2-positive invasive breast carcinoma treated with NACT in the years 2002-2014 and having achieved $\mathrm{pCR}$ was obtained from the patient database of the Peking University Cancer Hospital Breast Center. We included only unilateral non-metastatic tumors, excluding T4 and inflammatory tumors. Clinical and histopathological data were extracted from the database. The study was approved by the institutional review board with a waiver of patient informed consent and was performed in accordance with the 1964 Declaration of Helsinki and its later amendments.

Immunohistochemical stains for estrogen receptor (ER), progesterone receptor (PR), and human epidermal growth factor re-
Table 1. Patient and tumor characteristics

\begin{tabular}{|c|c|c|c|}
\hline & \multirow{2}{*}{$\begin{array}{l}\text { Trastuzumab } \\
\text { group }(\mathrm{n}= \\
140) \\
\mathrm{n}(\%)\end{array}$} & \multirow{2}{*}{$\begin{array}{l}\text { No-trastu- } \\
\text { zumab group } \\
(\mathrm{n}=83) \\
\mathrm{n}(\%)\end{array}$} & \multirow[t]{2}{*}{$\mathrm{p}$} \\
\hline & & & \\
\hline Age & & & 0.794 \\
\hline$<50$ & $70(50)$ & $43(51.8)$ & \\
\hline$\geq 50$ & $70(50)$ & $40(48.2)$ & \\
\hline T stage & & & 0.241 \\
\hline $\mathrm{T} 1-2$ & $134(95.7)$ & $77(92.8)$ & \\
\hline T3 & $6(4.3)$ & $6(7.2)$ & \\
\hline Grade & & & 0.279 \\
\hline $1-2$ & $95(67.9)$ & $63(75.9)$ & \\
\hline 3 & $45(32.1)$ & $20(24.1)$ & \\
\hline Estrogen receptor & & & 0.393 \\
\hline Positive & $53(37.9)$ & $22(26.5)$ & \\
\hline Negative & $87(62.1)$ & $61(73.5)$ & \\
\hline Pretreatment LN status & & & 0.097 \\
\hline FNA or $\mathrm{CNB}+$ & $50(35.7)$ & $25(30.1)$ & \\
\hline SLNB+ & $19(13.6)$ & $6(7.2)$ & \\
\hline SLNB- & $59(42.1)$ & $37(44.6)$ & \\
\hline Unidentified & $12(8.6)$ & $15(18.1)$ & \\
\hline Surgery & & & 0.337 \\
\hline BCT & $51(36.4)$ & $25(30.1)$ & \\
\hline Mastectomy & $89(63.6)$ & $58(69.9)$ & \\
\hline ALND & & & 0.869 \\
\hline Yes & $76(54.3)$ & $46(55.4)$ & \\
\hline No & $64(45.7)$ & $37(44.6)$ & \\
\hline Chemotherapy regimen & & & $<0.001$ \\
\hline $\begin{array}{l}\text { Anthracycline only } \\
\text { or taxane only }\end{array}$ & $43(30.7)$ & $53(63.9)$ & \\
\hline $\begin{array}{l}\text { Anthracycline }+ \\
\text { taxane }\end{array}$ & $97(69.3)$ & $30(36.1)$ & \\
\hline Radiotherapy & & & 0.233 \\
\hline Yes & $62(44.3)$ & $30(36.1)$ & \\
\hline No & $78(55.7)$ & $53(63.9)$ & \\
\hline Residual DCIS & & & $<0.001$ \\
\hline Yes & $28(20)$ & $35(42.2)$ & \\
\hline No & $112(80)$ & $48(57.8)$ & \\
\hline Endocrine therapy & & & 0.774 \\
\hline Yes & $55(39.3)$ & $31(37.3)$ & \\
\hline No & $85(60.7)$ & $52(62.7)$ & \\
\hline Adjuvant chemotherapy & & & $<0.001$ \\
\hline Yes & $15(10.7)$ & $26(31.3)$ & \\
\hline No & $125(89.3)$ & $57(68.7)$ & \\
\hline
\end{tabular}

LN, Lymph node; FNA, fine needle aspirate; CNB, core needle biopsy; SLNB, sentinel lymph node biopsy; BCT, breast-conserving therapy; ALND, axillary lymph node dissection; DCIS, ductal carcinoma in situ. 
Table 2. Details of patients with recurrence-free survival (RFS) events

\begin{tabular}{cllllll}
\hline $\begin{array}{l}\text { Patient } \\
\text { number }\end{array}$ & Trastuzumab & $\begin{array}{l}\text { Hormone } \\
\text { receptor }\end{array}$ & $\begin{array}{l}\text { T } \\
\text { stage }\end{array}$ & $\begin{array}{l}\text { Preoperative lymph node } \\
\text { status }\end{array}$ & $\begin{array}{l}\text { RFS, } \\
\text { months }\end{array}$ & Detail of recurrence or cause of death \\
\hline 1 & yes & negative & 2 & SLNB positive & 49 & LR (ipsilateral chest wall) \\
2 & yes & negative & 2 & FNA positive & 12 & distant metastasis (brain) \\
3 & yes & negative & 3 & FNA positive & 14 & distant metastasis (brain) \\
4 & yes & negative & 2 & SLNB negative & 28 & distant metastasis (lung) \\
5 & yes & negative & 2 & FNA positive & 38 & secondary cancer (leukemia) \\
6 & no & negative & 2 & SLNB negative & 91 & CBC \\
7 & no & negative & 1 & FNA positive & 128 & CBC \\
8 & no & negative & 2 & SLNB negative & 47 & CBC \\
9 & no & negative & 3 & FNA positive & 15 & distant metastasis (liver) \\
10 & no & negative & 2 & SLNB negative & 16 & distant metastasis (lung) \\
11 & no & negative & 2 & FNA positive & 43 & IBTR + distant metastasis (contralateral axilla) \\
12 & no & negative & 2 & FNA positive & 34 & IBTR \\
13 & no & negative & 2 & SLNB negative & 26 & distant metastasis (liver and bone) \\
14 & no & negative & 1 & FNA positive & 23 & LR (ipsilateral chest wall) + RR (ipsilateral \\
& & negative & 2 & SLN visualization failure & 89 & distant metastasis (lung) \\
15 & no & negative & 1 & SLNB negative & 30 & RR (ipsilateral axilla) \\
16 & no & negative & 2 & clinically suspicious & 15 & cause of death unknown \\
17 & no & negative & 2 & SLNB positive & 44 & secondary cancer (gastric) \\
18 & no & & & &
\end{tabular}

SLNB, Sentinel lymph node biopsy; FNA, fine needle aspirate; LR, local recurrence; RR, regional recurrence; CBC, contralateral breast cancer; IBTR, ipsilateral breast tumor recurrence.

ceptor 2 (HER2) were performed at the time of the initial diagnosis. ER and PR status were considered positive if $\geq 10 \%$ of cells stained positive. HER2 was examined either with immunohistochemistry or fluorescence in situ hybridization (FISH). HER2 immunohistochemical results of 0 and $1+$ were considered negative. An immunohistochemical result of $3+$ was considered positive. Cases with a HER2 immunohistochemical result of $2+$ were considered positive only when unequivocally amplified by FISH.

\section{Treatment}

All patients received anthracycline-based or taxane-based or sequential anthracycline-taxane NACT regimens. Adjuvant and/ or neoadjuvant trastuzumab was added to our treatment recommendations in the past decade. Surgery was usually scheduled 2-3 weeks after the end of NACT. Additional adjuvant chemotherapy and adjuvant radiotherapy were at the discretion of the treating physician and radiologist. All patients who underwent breast-conserving surgery (BCS) received radiotherapy. Endocrine therapy (tamoxifen, aromatase inhibitors, and gonadotropin-releasing hormone agonists when indicated) was prescribed for all patients with hormone receptor-positive disease. Patients were followed up regularly.

pCR was defined as the absence of invasive carcinoma in the breast resection specimen and lymph nodes (ypT0/is ypN0) for patients who underwent breast surgery and axillary lymph node dissection (ALND). For patients whose ALNDs were omitted due to negative sentinel lymph node biopsy, pCR was defined as the absence of invasive carcinoma in the breast resection specimen (ypT0/is).

\section{Study Endpoint}

The primary endpoint was RFS, defined as the interval between surgery and documented disease recurrence, progression, or death from any cause. Distant disease-free survival (DDFS) was defined as the time from surgery to distant metastasis, and OS was defined as the time from surgery to death.

\section{Statistical Analysis}

Statistical analysis was performed using SPSS software version 22.0 (IBM Corp., Armonk, NY, USA). For comparison of means, independent sample t-test was performed. For comparison of proportions, chi-square test or Fisher's exact test was performed. A p value of $<0.05$ was considered significant.

Patients were divided into 2 treatment groups according to trastuzumab use (fig. 1). We used the Kaplan-Meier method with log-rank estimates to test the prognostic effect of trastuzumab use on RFS, DDFS, and OS. 5-year survival with 95\% confidence interval (CI) was calculated for each treatment group. Univariate and multivariate Cox proportional hazards models were performed to obtain hazard ratios (HRs) or adjusted HRs with 95\% CIs.

\section{Results}

A total of 223 HER2-positive breast cancer patients who achieved pCR after NACT were identified. The baseline characteristics of these patients are summarized in table 1.The median age of the patients was 49 years (range 25-72 years), 210 (94.2\%) patients had T1-2 tumors. 137 (61.4\%) patients were ER- and PR-negative. BCS and subsequent radiotherapy were performed in 76 (34.1\%) patients. ALND was performed in 122 patients. 
Table 3. Univariate and multivariate analyses by patient and treatment characteristics

\begin{tabular}{|c|c|c|c|c|}
\hline \multirow[t]{2}{*}{ Factors } & \multicolumn{2}{|c|}{ Univariate analysis } & \multicolumn{2}{|c|}{ Multivariate analysis } \\
\hline & HR (95\% CI) & $\mathrm{p}$ & HR (95\% CI) & $\mathrm{p}$ \\
\hline \multicolumn{5}{|l|}{ Age, years } \\
\hline$\geq 50$ & $0.72(0.28-1.84)$ & \multirow{2}{*}{0.493} & - & \multirow{2}{*}{-} \\
\hline$<50$ & 1 & & - & \\
\hline \multicolumn{5}{|l|}{ T stage } \\
\hline T 3 & $2.18(0.50-9.50)$ & \multirow{2}{*}{0.300} & - & \multirow[b]{2}{*}{-} \\
\hline T $1-2$ & 1 & & - & \\
\hline \multicolumn{5}{|l|}{ Grade } \\
\hline 3 & $1.65(0.64-4.28)$ & \multirow{2}{*}{0.300} & - & \multirow{2}{*}{-} \\
\hline $1-2$ & 1 & & - & \\
\hline \multicolumn{5}{|l|}{ Hormone receptor } \\
\hline Negative & - & \multirow{2}{*}{0.083} & - & \multirow[b]{2}{*}{-} \\
\hline Positive & 1 & & - & \\
\hline \multicolumn{5}{|l|}{ FNA } \\
\hline Positive & $1.63(0.64-4.15)$ & \multirow{2}{*}{0.308} & - & \multirow[b]{2}{*}{-} \\
\hline Other & 1 & & - & \\
\hline \multicolumn{5}{|l|}{ SLNB } \\
\hline Positive & $1.10(0.25-4.82)$ & \multirow{2}{*}{0.899} & - & \multirow{2}{*}{-} \\
\hline Other & 1 & & - & \\
\hline \multicolumn{5}{|l|}{ Surgery } \\
\hline BCT & $1.06(0.40-2.85)$ & \multirow{2}{*}{0.902} & - & \multirow[b]{2}{*}{-} \\
\hline Mastectomy & 1 & & - & \\
\hline \multicolumn{5}{|l|}{ Radiotherapy } \\
\hline Yes & $0.55(0.19-1.54)$ & & - & \\
\hline No & 1 & 0.253 & - & - \\
\hline ALND & & & & \\
\hline Yes & $2.05(0.72-5.80)$ & & - & \\
\hline No & 1 & 0.176 & _- & \\
\hline Anthracycline $+\mathrm{t}$ & & & & \\
\hline Yes & $0.77(0.30-1.93)$ & & - & \\
\hline No & 1 & 0.572 & - & - \\
\hline Residual DCIS & & & & \\
\hline Yes & $1.81(0.70-4.70)$ & & - & \\
\hline No & 1 & 0.221 & - & - \\
\hline Endocrine therap & & & & \\
\hline Yes & $0.09(0.01-0.66)$ & & $0.09(0.01-0.66)$ & \\
\hline No & 1 & 0.087 & 1 & 0.018 \\
\hline Adjuvant chemot & & & & \\
\hline Yes & $1.05(0.40-2.72)$ & 0928 & - & \\
\hline No & 1 & 0.928 & - & - \\
\hline Trastuzumab & & & & \\
\hline Yes & $0.31(0.11-0.88)$ & & $0.31(0.11-0.89)$ & \\
\hline No & 1 & 0.028 & 1 & 0.030 \\
\hline
\end{tabular}

HR, Hazard ratio; CI, confidence interval; FNA, fine needle aspirate; SLNB, sentinel lymph node biopsy; ALND, axillary lymph node dissection; DCIS, ductal carcinoma in situ; BCT, breast-conserving therapy.

A total of $83(37.2 \%)$ patients were treated with chemotherapy only, and $140(62.8 \%)$ patients were treated with chemotherapy plus trastuzumab. All trastuzumab treatment started during the neoadjuvant stage and lasted for 1 year. More patients in the no-trastuzumab group had residual ductal carcinoma in situ (DCIS) compared to the trastuzumab group ( 42.2 vs. $20 \%, \mathrm{p}<0.001$ ). More patients in the trastuzumab group received sequential anthracycline and taxane chemotherapy (69.3 vs. $36.1 \%, \mathrm{p}<0.001)$.
The median follow-up time was 67 months, and a total of 18 patients relapsed: 4 patients had locoregional recurrences (LRR); 1 patient had concurrent local recurrence and distant metastasis; 3 patients had contralateral breast cancer (CBC); 7 patients had distant metastases only; 2 patients died of second cancers (1 gastric cancer and 1 leukemia); and in 1 patient, the cause of death could not be determined from the records. Details of the recorded RFS events are summarized in table 2. 
The 5-year RFS estimates by patient and tumor characteristics are summarized in table 3. The 5-year RFS was 95.7\% (95\% CI 91.8-99.6\%) in the trastuzumab group $(\mathrm{n}=140)$ and $87.8 \%(95 \% \mathrm{CI} 80.7-94.9 \%)$ in the no-trastuzumab group $(\mathrm{n}=83)$ (HR 0.31, HR 95\% CI 0.11-0.88, $\mathrm{p}=$ 0.028). The Kaplan-Meier estimates of RFS stratified by trastuzumab are presented in figure 2. The 5-year DDFS was $97.8 \%$ (95\% CI 95.4-99.9\%) in the trastuzumab group and $95.1 \%$ (95\% CI 90.4-99.8\%) in the no-trastuzumab group (HR 0.43, 95\% CI 0.10-1.88, $\mathrm{p}=0.250$ ). The 5-year OS was $97.7 \%$ (95\% CI 95.2-99.9\%) in the trastuzumab group and $96.3 \%$ (95\% CI 92.2-99.9\%) in the no-trastuzumab group (HR 0.55, HR 95\% CI 0.12-2.47, $\mathrm{p}=0.432$ ).

On multivariate analysis, after adjustment for age, T stage, grade, preoperative lymph node status, neoadjuvant and adjuvant chemotherapy regimens, type of surgery and adjuvant radiation, endocrine therapy and trastuzumab were found to be associated with decreased risk of relapse ( $p=0.018$ and 0.030 , respectively) (table 3 ).

\section{Discussion}

There is ongoing discussion regarding the 'de-escalation' of anti-HER2 treatment for certain subgroups of HER2-positive breast cancer [6]. One of the questions that remains to be answered is whether patients whose tumors were completely eradicated by NACT should still receive 1 year of trastuzumab as standard treatment. After a median follow-up of 5.4 years, of the 68 pCR patients in the NOAH trial (45 with trastuzumab and 23 with chemotherapy alone), the HR for event-free survival between those with and without trastuzumab was 0.29 (95\% CI 0.11-0.78) suggesting that the addition of trastuzumab resulted not only in more patients achieving a pCR, but also in a different quality of pCR [4].

In the present study, we report our survival outcomes focusing on patients achieving a pCR after NACT with or without trastuzumab. Patients in the no-trastuzumab group were treated before 1-year-trastuzumab became standard of care in our institution or sequential trastuzumab was planned after completion of NACT and surgery (which we no longer use). However, after careful consideration of the pros and cons of 1-year adjuvant trastuzumab and based on the fact that their tumors were completely eradicated by NACT, some patients with HER2-positive disease chose to withhold trastuzumab.

Our results showed improved RFS in the trastuzumab group compared to the no-trastuzumab group (95.7 vs. $87.8 \%, \mathrm{HR}=0.31, \mathrm{p}=0.028)$. It is noteworthy that all 18 patients with RFS events had hormone receptor-negative tumors and, as a consequence, lacked endocrine therapy. This observation is in accordance with the increasing recognition that hormone receptor-positive and -negative

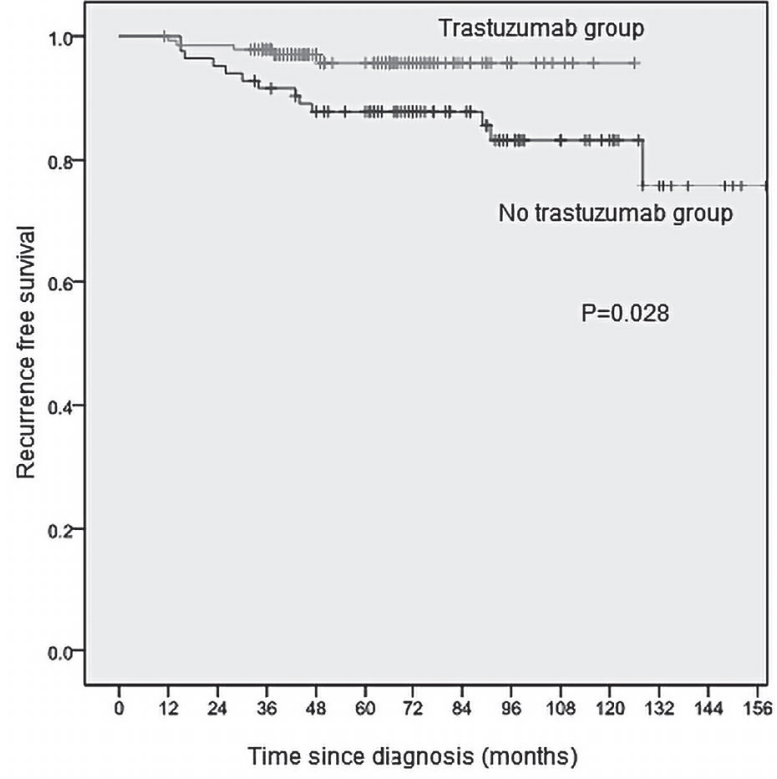

Fig. 2. Kaplan-Meier relapse-free survival (RFS) estimates.

breast cancers are different diseases [9]. Potential reasons for observed differences in RFS include cross-talk between the ER and HER2 pathways and the role of endocrine therapy in reducing the risk of relapse in patients with hormone receptor-positive disease. In addition, the use of endocrine therapy combined with HER2-targeted therapy represents a type of dual blockage for patients with both HER2-positive and hormone receptor-positive disease [10].

There were numerically less distant metastases in the trastuzumab group (3/140) compared to the no-trastuzumab group (5/83); however, no significant difference in DDFS or OS was observed between the 2 groups. The number of patients with LRR and CBC, respectively, was also lower in the trastuzumab group in our study (LRR $1 / 140$ vs. $4 / 83$, CBC $0 / 140$ vs. $3 / 83$ ). The effect of systemic trastuzumab on locoregional control and CBC needs further evaluation.

In the no-trastuzumab group, the most common sites of distant metastasis were liver and lung/pleura (4/5 patients), while in the trastuzumab group, brain metastasis is more common ( $2 / 3$ patients). It was reported that patients with HER2-positive breast cancer treated with trastuzumab live longer due to extracranial disease control and will experience central nervous system (CNS) metastasis more often $[11,12]$. In patients with an intact blood-brain barrier, trastuzumab is thought to have limited access to the brain because of its relatively large molecular size, and the CNS may represent a potential sanctuary site in patients with HER2-positive disease treated with trastuzumab. 
Most of the modern neoadjuvant trials incorporate 'no residual invasive tumor' as the definition of pCR (ypT0/ is ypN0). However, a German pooled analysis of 7 neoadjuvant trials showed that disease-free survival (DFS) was significantly superior in patients with no invasive and no in situ residuals in the breast or nodes compared with patients with residual DCIS [13]. In our study, more patients in the no-trastuzumab group had residual DCIS (42.2 vs. $20.0 \%, p<0.001$ ), but the presence of residual DCIS was not associated with a worse RFS in subsequent univariate or multivariate analysis.

In the N9831 trial, there was an increase in DFS with concurrent administration of trastuzumab and paclitaxel (HR 0.77), but the $\mathrm{p}$ value (0.02) did not cross the prespecified O'Brien-Fleming boundary (0.00116) for the interim analysis [14]. Sequential trastuzumab after completion of NACT and surgery is still a widely adopted method throughout the world. Therefore, one of the most important implications of the present study is that for HER2-positive patients who achieved pCR after NACT, the administration of adjuvant trastuzumab should be considered standard treatment. Furthermore, a hormone receptor-negative patient is at increased risk of relapse even after a pCR has been achieved. For patients with hormone receptor-negative and HER2-positive diseases, new treatment strategies are needed.

Our study has limitations. First and foremost, its retrospective nature makes it prone to biases. The chemotherapy regimens were not well balanced between the 2 groups; more patients in the trastuzumab group received sequential anthracycline and taxane chemotherapy. It is unlikely that this imbalance affected our result since it is a common belief that pCR patients have excellent survival and there is little or no room for further improvement. Secondly, all patients in the trastuzumab group received neoadjuvant trastuzumab, and preoperative trastuzumab is a confounding factor. None of our patients were treated with adjuvant trastuzumab only, prohibiting a comparison to the no-trastuzumab group. Thirdly, the limited number of patients and follow-up years makes it impossible to draw a definitive conclusion or perform further subgroup analysis. Still, to our knowledge, this is the largest group of HER2-positive patients who achieved pCR after NACT. Future meta-analysis of this subgroup of patients may be valuable.

Although preliminary and needing confirmation, we believe that our data provides an important additional piece of information for the treatment of HER2-positive breast cancer. Administration of adjuvant trastuzumab should be considered standard treatment even for HER2positive patients who achieved pCR after NACT.

\section{Acknowledgment}

We would like to thank Dr. John Yim, City of Hope National Medical Center, for his assistance in preparing this manuscript.

\section{Disclosure Statement}

The authors declare that they have no conflict of interest.

\section{References}

1 Shah MV, Wiktor AE, Meyer RG, et al: Change in pattern of HER2 fluorescent in situ hybridization (FISH) results in breast cancers submitted for FISH testing: experience of a reference laboratory using US Food and Drug Administration criteria and American Society of Clinical Oncology and College of American Pathologists guidelines. J Clin Oncol 2016;34:3502-3510.

2 Buzdar AU, Valero V, Ibrahim NK, et al: Neoadjuvant therapy with paclitaxel followed by 5-fluorouracil, epirubicin and cyclophosphamide chemotherapy and concurrent trastuzumab inhuman epidermal growth factor receptor 2-positive operable breast cancer: an update of the initial randomized study population and data of additional patients treated with the same regimen. Clin Cancer Res 2007;13:228-233.

-3 Untch M, Fasching PA, Konecny GE, et al Pathologic complete response after neoadjuvant chemotherapy plus trastuzumab predicts favorable survival in human epidermal growth factor receptor 2-overexpressing breast cancer: results from the TECHNO tria of the AGO and GBG study groups. J Clin Oncol 2011;29:3351-3357.
-4 Gianni L, Eiermann W, Semiglazov V, et al: Neoadjuvant and adjuvant rastuzumab in patients with HER2-positive locally advanced breast cancer (NOAH): follow-up of a randomized controlled superiority trial with a parallel HER2-negative cohort. Lancet Oncol 2014; 15:640-647.

5 Cortazar P, Zhang L, Untch M, et al: Pathological complete response and long-term clinical benefit in breast cancer: the CTNeoBC pooled analysis. Lancet 2014;384:164-172.

$\checkmark 6$ Joensuu H: Escalating and de-escalating treatment in HER2-positive early breast cancer. Cancer Treat Rev 2017;52:1-11.

7 Gonzalez-Angulo AM, Parinyanitikul N, Lei $\mathrm{X}$, et al: Effect of adjuvant trastuzumab among patients treated with anti-HER2-based neoadjuvant therapy. Br J Cancer 2015;112:630635.

8 Carey LA, Dees EC, Sawyer L, et al: The triple negative paradox: primary tumor chemosensitivity of breast cancer subtypes. Clin Cancer Res 2007;13:2329-2334.

-9 Ellis MJ: HER2-positive breast cancer, intrinsic subtypes, and tailoring therapy. J Natl Cancer Inst 2014;106:dju212.
10 O'Sullivan CC, Bradbury I, Campbell C, et al: Efficacy of adjuvant trastuzumab for patients with human epidermal growth factor receptor 2 -positive early breast cancer and tumors $\leq 2$ $\mathrm{cm}$ : a meta-analysis of the randomized trastuzumab trials. J Clin Oncol 2015;33:26002608.

11 Musolino A, Ciccolallo L, Panebianco M, et al: Multifactorial central nervous system recurrence susceptibility in patients with HER2positive breast cancer: epidemiological and clinical data from a population-based cancer registry study. Cancer 2011;117:1837-1846.

12 Mehta AI, Brufsky AM, Sampson JH: Therapeutic approaches for HER2-positive brain metastases: circumventing the blood-brain barrier. Cancer Treat Rev 2013;39:261-269.

13 Von Minckwitz G, Untch M, Blohmer JU, et al: Definition and impact of pathologic complete response on prognosis after neoadjuvant chemotherapy in various intrinsic breast cancer subtypes. J Clin Oncol 2012;30:1796-1804.

14 Perez EA, Suman VJ, Davidson NE, et al: Sequential versus concurrent trastuzumab in adjuvant chemotherapy for breast cancer. J Clin Oncol 2011;29:4491-4497. 